\title{
Synthesis of ZnO Crystals Hexagonal Ball Shape
}

\author{
A. Medina-Flores ${ }^{1}$, S. E. Borjas-García ${ }^{2}$, L. Béjar ${ }^{1}$, P. Martínez-Torres ${ }^{2}$, J. L. Bernal ${ }^{3}$, C. Aguilar ${ }^{4}$ \\ ${ }^{1}$ Instituto de Investigaciones Metalúrgicas, Universidad Michoacana de San Nicolás de Hidalgo, \\ Morelia, Michoacán, México \\ ${ }^{2}$ Instituto de Física y Matemáticas, Universidad Michoacana de San Nicolás de Hidalgo, Morelia, \\ Michoacán, México \\ ${ }^{3}$ Automotive Mechanics Department. Universidad Politécnica de Pachuca. Zempoala, Hidalgo. México \\ ${ }^{4}$ Universidad Técnica Federico Santa Maria. Valparaiso Chile
}

Zinc oxide is an important material due to its many applications. It is a wide band gap semiconductor that is piezoelectric and has catalytic activity [1,2]. These properties make it useful in a variety of applications such as solar cells, piezoelectric and chemical sensors [3]. The crystal structure and macroscopic morphology of the $\mathrm{ZnO}$ crystals dictates their properties and, thus, the applications for which they can be used. The ability to tailor both the crystal structure and morphology would allow $\mathrm{ZnO}$ crystals to be tuned for specific applications. Based on the reaction conditions used in the synthesis of $\mathrm{ZnO}$ structures, nanorods, nanowires, nanorings, nanobelts and nanosprings, among others, can be formed [2]. ZnO ball crystal shape was sinthetized using both Sol - Gel method with a hydrothermal soft treatment. In a first step, two solutions were prepared. The first one was obtained by dissolving $5.9498 \mathrm{~g}$ of $\mathrm{Zn}\left(\mathrm{NO}_{3}\right)_{2} .6 \mathrm{H} 2 \mathrm{O}$ in $21.618 \mathrm{~g}$ distilled water. For the second solution, $7.999 \mathrm{~g}$ of $\mathrm{NaOH}$ was dissolved in $21.618 \mathrm{~g}$ distilled water. In a second step, the Na-solution was added slowly (drop by drop) to $\mathrm{Zn}$-solution and stirred at room temperature. The final solution was stirred for $30 \mathrm{~min}$ at room temperature to get a material with a molar ratio of $\mathrm{Zinc}: \mathrm{NaOH}: \mathrm{H} 2 \mathrm{O}=1: 10: 120$. The gel obtained was aged in a stainless steel autoclave bottle at $160{ }^{\circ} \mathrm{C}$ for 1 day. After the hydrothermal treatment, the sample was washed with $100 \mathrm{ml}$ of distilled water and centrifuged at $12000 \mathrm{rpm}$ during $10 \mathrm{~min}$. After that, the material was dried at room temperature for 1 day. The surface morphology of the samples was analyzed by using a scanning electron microscopy FEG-SEM JEOL JSM 7600. Figure 1 shows an SEM image of several $\mathrm{ZnO}$ Crystal hexagonal ball shape showing a diameter about $10 \mathrm{~nm}$ and the formation of hexagonal bars with diameters ranged between 1 to $5 \mu \mathrm{m}$ and length from 3 to $15 \mu \mathrm{m}$. Figure 2 shows a zoom of a $\mathrm{ZnO}$ crystal hexagonal shape from figure 1 where it is possible to appreciate the form of the $\mathrm{ZnO}$ crystal. The results showed that the molar ratio $\mathrm{Zn}\left(\mathrm{NO}_{3}\right)_{2} .6 \mathrm{H} 2 \mathrm{O} / \mathrm{NaOH}$ and hydrothermal treatment temperature could be critical parameters in the formation of hexagonal ball shape. The high amount of $\mathrm{NaOH}$ in the synthesis could increase drastically the $\mathrm{pH}$ during the formation of the gel. This effect could be the reason for the formation of several hexagonal bar to form a ball shape. The synthesis procedure presented in this research work is easy with a low cost and can be used to synthetize different kinds of material.

\section{References}

[1] Look D. C. Mater Sci Eng B Volume 7. (2001) p. 383

[2] Fan Z et al, J. nanosci. Nanotechnol Volume 5 (2005) p. 1561.

[3] Gordillo G. Surf Rev Lett Volume 9 (2002) p. 1675. 


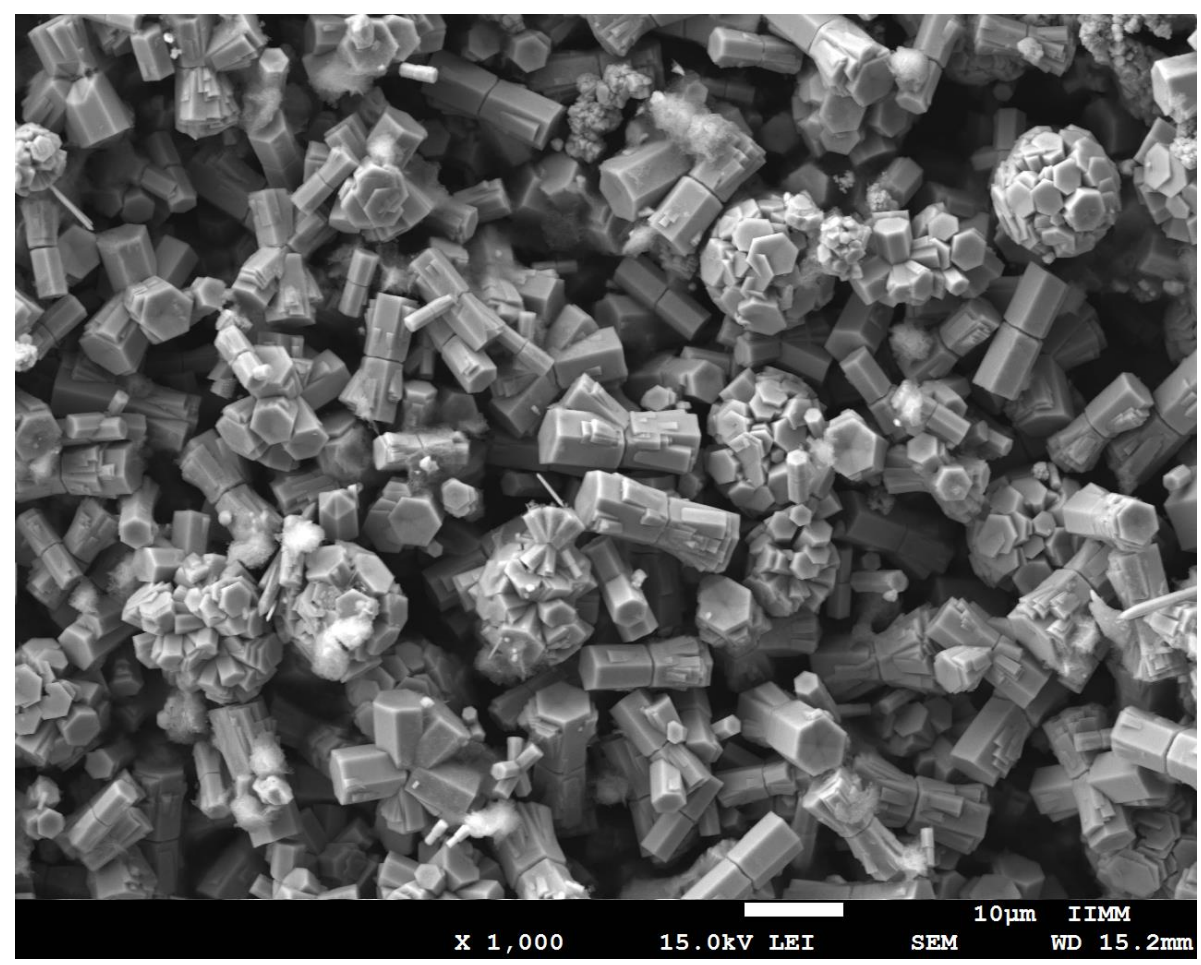

Figure 1. SEM image of $\mathrm{ZnO}$ crystals hexagonal balls shape

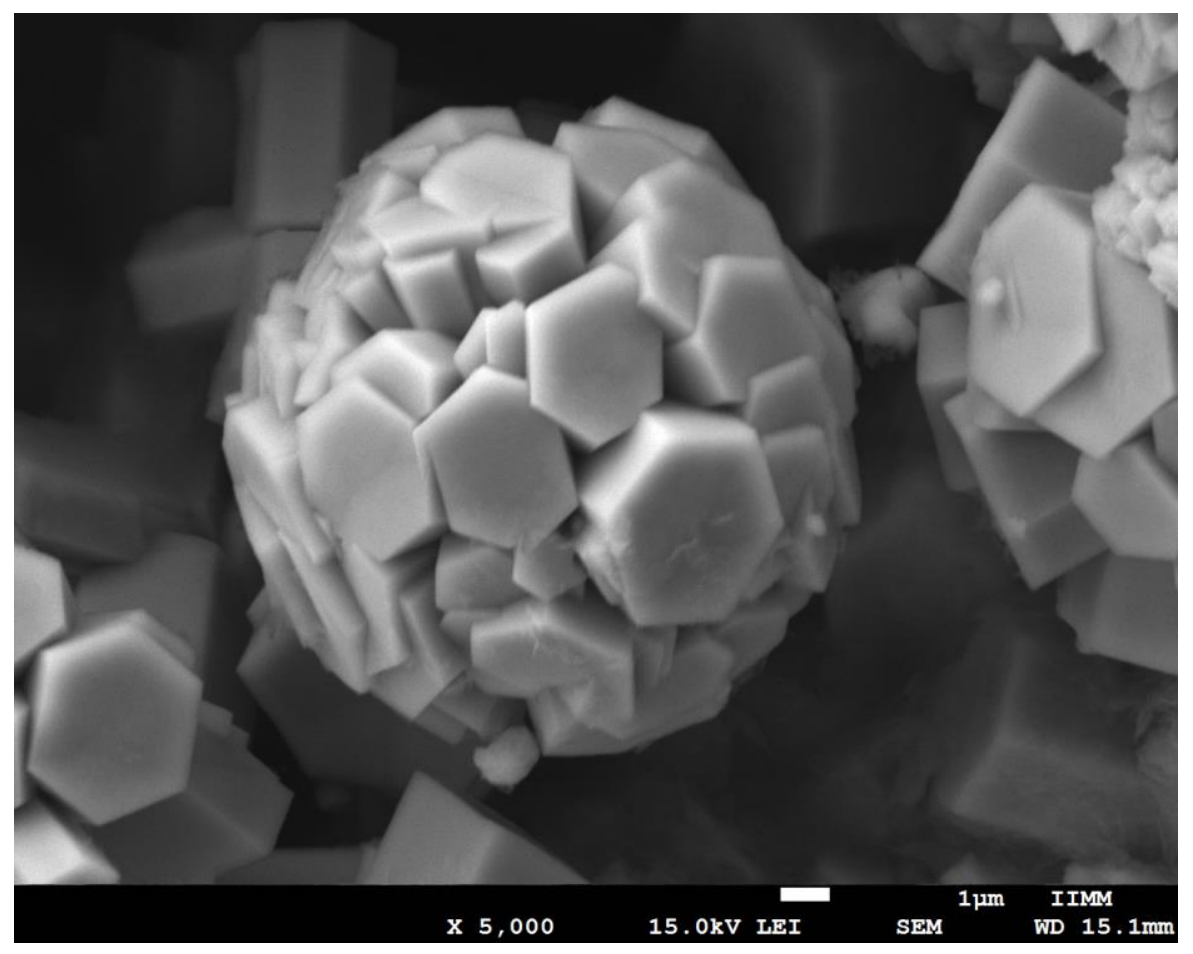

Figure 2. Zoom image of a one $\mathrm{ZnO}$ crystal hexagonal ball shape from figure 1 . 